\title{
The impact of metallothionein-II on microglial response to tumor necrosis factor-alpha (TNFa) and downstream effects on neuronal regeneration
}

\author{
Jacqueline Y. K. Leung ${ }^{1 *}$ (D, William R. Bennett ${ }^{1}$, Anna E. King ${ }^{1}$ and Roger S. Chung ${ }^{2}$
}

\begin{abstract}
Background: The extracellular environment plays an important role in supporting the regeneration of axons after injury. Metallothionein-II (MTII) is a metal-binding protein known for its neuroprotective effect by directly stimulating the growth of axons after injury. Previous studies have shown that MTII also modulates the response of astrocytes and microglia after injury. However, a detailed analysis describing how MTII modulates the interaction between microglia and neurons is lacking.

Methods: We introduced fluorescently labelled MTII into the cortex at the time of needlestick injury to investigate the cellular uptake of MTII using immunohistochemistry with antibodies against cell-type-specific markers. The role of MTII in modulating the effect of microglia on axon outgrowth following an inflammatory response is further investigated using a co-culture model involving primary rodent microglia pre-treated with TNFa and primary rodent cortical neurons. The axon lengths were assessed $24 \mathrm{~h}$ after the plating of the neurons onto treated microglia. We also utilised siRNA to knockdown the expression of LRP1, which allows us to investigate the role of LRP1 receptors in the MTII-mediated effect of microglia on axon outgrowth.
\end{abstract}

Results: Fluorescently labelled MTII was found to be associated with neurons, astrocytes and microglia following injury in vivo. Microglia-neuron co-culture experiments demonstrated that exogenous MTII altered the response of microglia to TNFa. The neurons plated onto the TNFa-stimulated microglia pre-treated with MTII have shown a significantly longer axonal length compare to the TNFa-stimulated microglia without the MTII treatment. This suggested that MTII reduce cytokine-stimulated activation of microglia, which would ordinarily impair neurite outgrowth. This inhibitory effect of MTII on activated microglia was blocked by siRNA-mediated downregulation of LRP1 receptor expression in microglia, suggesting that MTII acts via the LRP1 receptor on microglia.

Conclusions: This study demonstrates that exogenous MTII acts via the LRP1 receptor to alter the inflammatory response of microglia following TNFa stimulation, providing a more supportive environment for axon growth.

Keywords: Metallothionein, Microglia, Low-density lipoprotein receptor-related protein-1, Low-density lipoprotein receptorrelated protein-2, Axon regeneration, Traumatic brain injury

\footnotetext{
* Correspondence: Jacqueline.Leung@utas.edu.au

${ }^{1}$ Wicking Dementia Research and Education Centre, College of Health and Medicine, University of Tasmania, Medical Science Precinct 1, 17 Liverpool Street, Hobart, TAS 7000, Australia

Full list of author information is available at the end of the article
} 


\section{Background}

Glial cells play an important role in maintaining the health of neurons and particularly following injury to the central nervous system (CNS). In this regard, astrocytes and microglia contribute actively to the formation of the glial scar, which is strongly inhibitory to axon regeneration $[1,2]$. Chondroitin sulphate proteoglycans (CSPGs) are one of the major molecules expressed by glia that contribute to the inhibitory environment formed by the glial scar. Modulating this inhibitory environment using agents such as chondroitinase $\mathrm{ABC}$ has been shown to improve the regeneration outcome after injury [3]. Chondroitinase ABC enzymatically digests CSPG, which improves neuron survival, axonal growth as well as synaptic plasticity following middle cerebral artery occlusion [4]. These, and many other studies [5-7], demonstrate that improving the extracellular environment plays a vital role in improving axon regeneration after injury. Apart from CSPG, myelinassociated inhibitors such as myelin-associated glycoprotein (MAG), Nogo and Oligodendrocyte myelin glycoprotein (OMgp) are also present in the glial environment and have a significant role in inhibiting axon outgrowth as well as plasticity $[6,8,9,10]$. Soluble inhibitory cues such as semaphorins are also recognised as having an important contribution to the inhibitory environment after CNS injury $[9,10]$. In contrast to factors that inhibit neurite outgrowth, other factors have been identified to promote neurite outgrowth. These include neurotrophic factors (such as nerve growth factors, brain-derived neurotrophic factor) as well as several extracellular matrix attractive guidance molecules that are present at the injury site $[6,9,10]$. The balance between the inhibitory and promoting factors in the injury site are important in determining the outcome of axon regeneration after injury.

Metallothionein I/II represents an unusual family of metal-binding proteins; both MTI and MTII isoform share a very similar structure as well as have a range of neurotrophic properties. Our group has previously demonstrated that extracellular MTII directly interacts with axons and improves axon outgrowth via the low-density lipoprotein receptor 2 (LRP2, also called megalin) [9, 10]. Apart from its direct actions on neurons, we have also identified a potential role of MTI/II in modulating the trophic environment provided by the glial response to promote neuron regeneration $[11,12]$. The use of transgenic animals (both MTI/II knockout and MTI overexpression mice) has further demonstrated that MTI/II has an active role in modulating the microglial response after injury. When a freeze-lesion cortical injury (a localised cortical injury caused by applying dry ice directly on the skull) was performed on the mice with MTI overexpression, this results in a significant decrease in the number of activated microglia near the freeze lesion site in comparison to the wild-type control mice [13]. In contrast, the freeze-lesion cortical injury in MTI/II-knockout mice results in an increase in microglia/macrophage infiltration and activation as well as a delayed in wound repair process when compared to the wild-type control mice [14]. Similar results have also been reported in rodent needlestick injury model, where the administration of extracellular MTII into the injury site improved regenerative sprouting of axons [15].

Together, these studies suggested that both MTI and MTII have an active role in modulating the inflammatory response as well as wound healing after injury. Mechanistic studies performed in our laboratory have now begun to reveal how MTII modulates the neuroinflammatory response of microglia following traumatic brain injury. We have previously shown that MTII has a direct effect in decreasing the expression of quinolinic acid (QUIN) in microglia along the injury tract in the rat cortical needlestick injury model [12]. QUIN is one of the neurotoxic intermediates of the kynurenine pathway that is significantly upregulated in inflammatory microglia and macrophages [16]. In cultured microglia, MTII treatment led to a decrease in QUIN expression upon stimulation with the pro-inflammatory interferon- $\gamma$ (IFN- $\gamma$ ) [12]. In the current study, we have explored the mechanism of MTII signalling in microglia and the subsequent impact upon neuronal regeneration. We show that MT modulates the inflammatory response of tumor necrosis factor (TNF)- $\alpha$-treated microglia via the LRP1 receptor and that this action of MTII upon microglia causes them to become a more permissive substrate to support axonal outgrowth in vitro.

\section{Methods}

\section{Metallothioneinll protein labelling}

The MTII protein used in these studies was native rabbit $\mathrm{Zn}_{7}$-MT-IIA (the major brain-expressed mammalian MTII isoform) purified by high-performance liquid chromatography (Bestenbalt LLC, Estonia). The MTII was N-terminally tagged using the AlexaFluor 488 Protein Labelling Kit (Invitrogen) in accordance with the accompanying protocols. During the column fractionation of labelled protein, all of the eluted fractions were collected including the unincorporated dye (the second eluted fluorescent peak). The fractions collected from the first eluted fluorescent peak contained the MTII conjugated with the AlexaFluor 488 tag and is hereafter referred to as $\mathrm{MT}_{488}$, while the unincorporated dye peak was used as the vehicle treatment and is referred to as $\mathrm{A}_{488}$.

\section{Focal cortical needlestick injury in adult rats}

Focal needlestick injuries to the Par1 somatosensory region of the cortex were performed as described previously [15]. Briefly, a $1 \mu \mathrm{L}$ 25-gauge Hamilton syringe was used to stereotactically deliver the different treatments: $\mathrm{MT}_{488}(2 \mathrm{mg} / \mathrm{mL})$ and $\mathrm{A}_{488}$ into the cortex $(n=4$ per 
treatment). The volume of injection for all treatments was $1 \mu \mathrm{L}$. The injury was induced by inserting the Hamilton syringe to a depth of $1.5 \mathrm{~mm}$ into the cortex. After leaving the syringe in place for $5 \mathrm{~min}, 0.5 \mu \mathrm{L}$ of the treatment was injected into the injury site. After a further $5 \mathrm{~min}$, the needle was first raised by $0.5 \mathrm{~mm}$ and the remaining $0.5 \mu \mathrm{L}$ of the treatment was then injected into the cortex. Absorbent Gelfoam (soaked with $5 \mu \mathrm{L}$ of the treatment solution, either $\mathrm{MT}_{488}$ or $\mathrm{A}_{488}$ ) was placed on the surface of the cortex, and the site of injury was sutured closed.

\section{Perfusion and processing of the brain injury site}

The animals were sacrificed 4 days after injury by an intraperitoneal injection of pentobarbitone $(0.4 \mathrm{mg} / \mathrm{g}$ of animal weight) to induce a deep anaesthesia followed by cardiac perfusion with $4 \%$ paraformaldehyde. The brain was then excised and post-fixed in $4 \%$ paraformaldehyde $+4 \%$ sucrose solution in phosphate buffer saline (PBS) for $24 \mathrm{~h}$. Brains were cryoprotected in 10 and $30 \%$ sucrose solutions $\left(24 \mathrm{~h}\right.$ incubation at $4{ }^{\circ} \mathrm{C}$ for each solution) prior to cryosectioning. The brain samples were cryosectioned at $16 \mu \mathrm{m}$ in a horizontally sectioned plane cut parallel to the superior surface of the brain using the Leica CM1850 Cryostat, and serial sections were then mounted on APTS-treated microscopic slides. For a consistent comparison between treatments, the sections were obtained at similar depth across all treatments. Sections that were collected and processed for immunohistochemistry labelling were located from approximately 60 to $540 \mu \mathrm{m}$ from the top of the cortex, corresponding to layer II and III of the supragranular layer of the somatosensory cortex.

\section{Immunohistochemistry on tissue sections}

Brain sections were incubated with the following primary antibodies: Mouse (ms)-SMI312 (1:1000, Covance), ms-MT (1:1000, Dako), Rabbit (rb)-Iba-1 (1:500, Wako), Rb- Glial fibrillary acidic protein (GFAP, 1:1000, Dako). All primary antibodies used were diluted in $0.03 \%$ Triton-X/PBS and incubated at $4{ }^{\circ} \mathrm{C}$ overnight. Following the incubation, the primary antibodies were washed three times with PBS. The sections were then incubated with the respective secondary antibodies: AlexaFluor594 or -488 anti-mouse (1:1000, Invitrogen), AlexaFluor594 or -488 anti-rabbit (1:1000, Invitrogen). The secondary antibodies were diluted in PBS and applied to sections for $1 \mathrm{~h}$ at room temperature prior to incubation with Nuclear Yellow (1 $\mu \mathrm{g} / \mathrm{mL}$, Molecular Probes) for $5 \mathrm{~min}$. The sections were mounted using fluorescent mounting agent (Dako).

\section{Image acquisition}

Images were captured using either an epifluorescence microscope (Olympus BX50) with mounted camera (CoolSNAP $\mathrm{HQ}^{2}$ camera) or confocal microscope (Zeiss LSM
510). In some cases, a confocal $Z$-axis series of digital images was captured through the injury site (minimum $0.5 \mu \mathrm{m} Z$-axis spacing).

\section{Purified rodent microglia cultures}

Purified rodent microglia cultures were prepared as described previously [12]. Briefly, mixed glial cultures were prepared from the cortex of Sprague Dawley postnatal day 2 rat pups in $75 \mathrm{~cm}^{2}$ tissue culture flasks, and maintained at $37{ }^{\circ} \mathrm{C}$ with $5 \% \mathrm{CO}_{2}$. The culture medium (Dulbecco's modified eagle medium, DMEM, from Sigma $+10 \%$ Fetal calf serum from Gibco $+1 \%$ penicillin-streptomycinamphotericin B solution from Gibco) was replaced on the following day with fresh medium, and culture was maintained to confluence (around 2 weeks after initial plating). Upon confluence, the culture medium was changed to serum-free medium (DMEM $+1 \%$ penicillin-streptomycinamphotericin B solution), and flasks were horizontally shaken at $250 \mathrm{rpm}$ for $30 \mathrm{~min}$, which was sufficient to dislodge the microglia in these mixed glial cultures. The culture medium containing the microglia was collected, and microglia were pelleted by centrifugation at $500 \times g$ for $10 \mathrm{~min}$. Microglia were re-suspended and then plated at the required number into 24-well plates $(15,000$ cells per well containing coverslips pre-coated with $0.025 \%$ poly-Llysine). Microglia were maintained in serum-free media until confluence.

\section{Neuron and microglia co-culture}

Microglia were collected and plated onto glass coverslips in serum-free media as described previously. Two days after the plating of microglia, the cultures were incubated with the respective treatment accordingly for $24 \mathrm{~h}$ in serum-free media. These treatments were $10 \mathrm{ng} / \mathrm{mL}$ TNF $\alpha$ (Abcam) with or without MTII $(1 \mu \mathrm{g} / \mathrm{mL})$ and saline (vehicle treatment). Cortical neuron cultures were prepared as reported previously from embryonic day 17 Sprague-Dawley rats [15]. In order to prevent the effect of the extracellular MTII or TNF $\alpha$ on the growing neurons, the media of the microglia culture was changed to neuron media (Neurobasal ${ }^{\mathrm{Ts}}$ medium supplement with B-27 supplement, $0.1 \mathrm{mM}$ L-glutamine and $10 \%$ fetal bovine serum from Gibco) prior to the addition of cortical neurons on top of the microglia at cell density of $2 \times 10^{4}$ cells per well. Co-cultures were incubated for $24 \mathrm{~h}$ followed by fixation with $4 \%$ paraformaldehyde for $15 \mathrm{~min}$ at room temperature.

\section{siRNA knockdown of LRP1 receptors}

We then employed pre-designed siRNA (ThermoFisher Scientific) to selectively knockdown the LRP1 receptors in microglia cultures as described previously [17]. The knockdown of LRP1 receptors expression in microglia was also confirmed via Western blotting as described 
previously [17]. The microglia were first collected (as above) and resuspended in $1 \mathrm{~mL}$ of serum-free media containing $100 \mathrm{nM}$ of siRNA against LRP1 (from ThermoFisher Scientific). The microglia were plated into 24-well plates (procedure stated as above) and incubated for $1 \mathrm{~h}$ at $37{ }^{\circ} \mathrm{C} 5 \% \mathrm{CO}_{2}$. The concentration of the siRNA was diluted down to $10 \mathrm{nM}$ in the well by adding in the additional culture media; the cultures were then returned back to the incubator.

\section{Immunocytochemistry on culture}

Fixed cultures were incubated with primary antibodies (ms-SMI312 used at 1:1000, Covance; rb-LRP1 used at 1:1000, Sigma-Aldrich; rb-megalin used at 1:1000, Santa Cruz Biotechnology) diluted in 0.03\% Triton-X/PBS for $24 \mathrm{~h}$ at $4{ }^{\circ} \mathrm{C}$. The primary antibodies were then washed three times in PBS (5 min per washes on shaker at room temperature). The coverslips were incubated with secondary antibodies (AlexaFluor-488 anti-mouse used at 1:1000, Invitrogen) in PBS for $1 \mathrm{~h}$ at room temperature on a shaker. All cultures were labelled with Nuclear Yellow $(1 \mu \mathrm{g} / \mathrm{mL}, 5 \mathrm{~min})$ followed by two times washes with PBS. Cultures were mounted on glass slides using fluorescent mounting agent (Dako).

\section{Imaging and statistical analysis}

Digital images (five images per coverslips) of SMI312 immunolabelled axons were acquired on a fluorescence microscope (Leica DM-LB2) with an Olympus Magnifier cooled-CCD camera. Lengths of axons were measured using ImageJ (National Institute of Health) software. The data were analysed using the Student $t$ test, with $p<0.05$ considered as significant. For MTII and TNF $\alpha$ experiment, $n=4$ experimental cultures were prepared and one coverslip per culture per treatment group was used. For LRP1 siRNA experiment, $n=4$ experimental cultures were prepared and two coverslips per culture per treatment group were used.

\section{Results}

Exogenous metallothionein-II is associated with neuron, astrocytes and microglia after needlestick injury

Our previous studies have shown that MTII can promote neurite outgrowth of cultured cortical neurons [15], through a mechanism involving LRP receptors and downstream MAPK signalling $[9,10]$. We have also shown in cell culture that MTII can modulate the astrocytic response to neurotrauma, leading to a more permissive environment to allow the extension of axons [11]. Similarly, MTII can act upon cultured microglia to suppress their inflammatory activation following cytokine stimulation. When administered by direct injection into the site of a cortical needlestick injury, MTII modulates glial reactivity and promotes regenerative axonal sprouting [15]. What remains unclear is which cell type/s are directly modulated by MTII treatment in vivo and the sequence of cellular events elicited by MTII that ultimately contributes to improved neural regeneration. To investigate this, we utilised fluorescently labelled MTII $\left(\mathrm{MT}_{488}\right)$, which was introduced into the cortex via the needlestick injury. Tissue was harvested at 4 days post-injury prior to immunohistochemistry.

We first confirmed that the administration of $\mathrm{MT}_{488}$ produces the same neuroprotective effect that has been reported previously. Accordingly, it was found that $\mathrm{MT}_{488}$ reduced the severity of the experimental injury, clearly observable by immunohistochemistry of axonal structures (using antibody against SMI312) (results not shown). This demonstrates that the $\mathrm{MT}_{488}$ displays similar neurotrophic activity to unlabelled MTII [15].

We characterised the cells that are associated with the administered $\mathrm{MT}_{488}$ after injury by performing immunofluorescent labelling using markers for neurons (SMI312, phosphorylated neurofilament), astrocytes (GFAP) and microglia (Iba-1) (Fig. 1). We show that $\mathrm{MT}_{488}$ was associated with neurons (particularly prevalent in axonal structures labelled with SMI312; Fig. 1a), microglia and astrocytes (labelled with Iba-1 and GFAP; Fig. 1d, g). In control tissue injected with $\mathrm{A}_{488}$, none of the neurons and astrocytes was associated with the injected $\mathrm{A}_{488}$; however, the $\mathrm{A}_{488}$ was shown to be associated with the Iba-1 positive microglia (Fig. 1g). Notably, microglia that had associated with $\mathrm{MT}_{488}$ displayed a ramified morphology, while those associating with $\mathrm{A}_{488}$ displayed a rounded (or amoeboid) morphology. We also observed that the majority of the $\mathrm{MT}_{488}$ was located within cellular structures surrounding the lesion site. In contrast, $\mathrm{A}_{488}$ was mainly located within the lesion site along with the cells debris.

\section{Role of metallothionein-II in modifying microglial response after injury}

Given that the uptake of $\mathrm{MT}_{488}$ was observed within microglia and that this was associated with an altered cellular morphology, we next examined the effect of exogenous MTII upon reactive microglia, using an in vitro microglia and neuron co-culture model. Firstly, we established the effect of activated microglia (TNF $\alpha$ stimulated) upon neurite outgrowth of co-cultured cortical neurons. Measurement of axonal extension $24 \mathrm{~h}$ after the plating of cortical neurons demonstrated that axons grown on activated microglia (TNF $\alpha$-treated) were significantly $(p<0.05)$ shorter than axons grown on microglia treated with saline (vehicle) (Fig. 2a, b). When the axons were plated on microglia that were pre-treated with TNF $\alpha$ and MTII (Fig. 2c), the length of the axons was significantly longer than neurons treated with TNF $\alpha$ only and not significantly different to the salinetreated microglia (Fig. 2d). This demonstrated that the addition of MTII on TNF $\alpha$-treated microglia ameliorated the inhibitory activity of TNF $\alpha$-treated microglia, restoring 

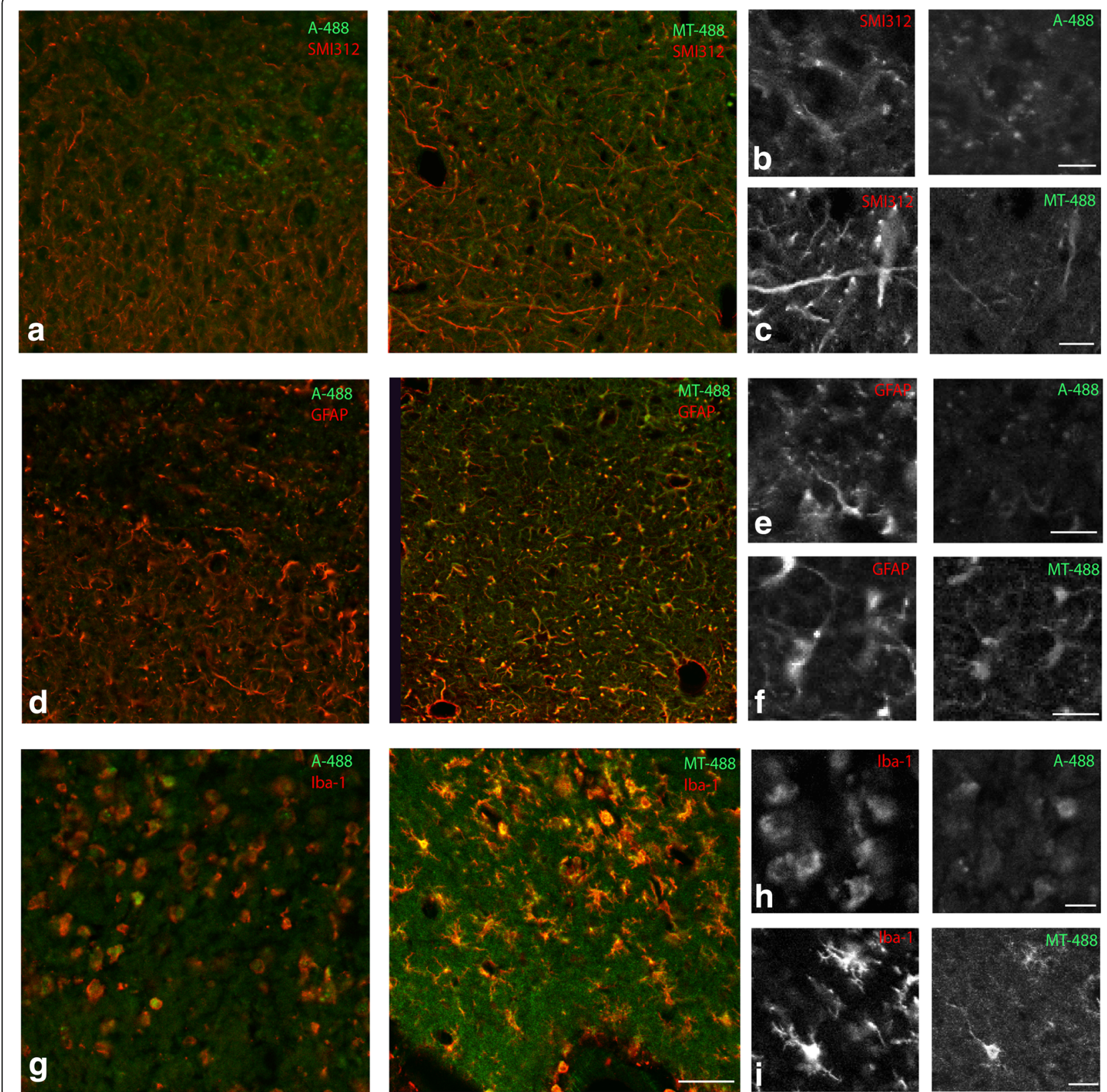

Fig. 1 Fluorescently labelled Metallothionein I/II (MT $\left.{ }_{488}\right)$ is taken up by neurons, astrocytes and microglia after needlestick injury. Immunohistochemistry using markers to label the neurons (SMI312), astrocytes (GFAP) and microglia (Iba-1) on frozen cortical sections collected from adult rat 4 days after receiving needlestick injury with either the $\mathrm{A}_{488}$ or $\mathrm{MT}_{488}$. The $\mathrm{MT}_{488}$, but not $\mathrm{A}_{488}$, was visible in neurons (a); higher magnification images shown in $\mathbf{b}$ and c, similarly, the astrocytes also appeared to take up the $\mathrm{MT}_{488}$ but not $\mathrm{A}_{488}(\mathbf{d})$; higher magnification images shown in $\mathbf{e}$ and $\mathbf{f}$. In contrast, both $\mathrm{A}_{488}$ and $\mathrm{MT}_{488}$ were taken up by microglia $(\mathbf{g})$; higher magnification images shown in $\mathbf{h}$ and $\mathbf{i}$. The microglia that taken up $\mathrm{A}_{488}$ appear in a more amoeboid-like morphology when compared to the microglia receiving the $\mathrm{MT}_{488}$, which had a more ramified phenotype. (Scale bar in $\mathbf{a}, \mathbf{d}$ and $\mathbf{g}=50 \mu \mathrm{m} ; \mathbf{b}, \mathbf{c}, \mathbf{e}, \mathbf{g}, \mathbf{h}$ and $\mathbf{i}=12 \mu \mathrm{m})$

neurite outgrowth to a level similar to that in cells receiving the vehicle treatment (Fig. 2).

\section{Metallothionein-II acts through LRP1 receptors in microglia}

We have previously demonstrated that MTII acts through the LRP2 (or megalin) receptor on neurons to improve regenerative sprouting of injured neurons $[9,10]$. Here, we determined if LRP receptors are also involved in the action of MTII upon cultured microglia. We firstly characterised the LRP expression in cultured microglia, and we observed a robust immunolabelling of LRP1 primarily present in the cell bodies (Fig. 3a). In contrast, no LRP2 labelling was observed in the cultured microglia (Fig. 3b). 

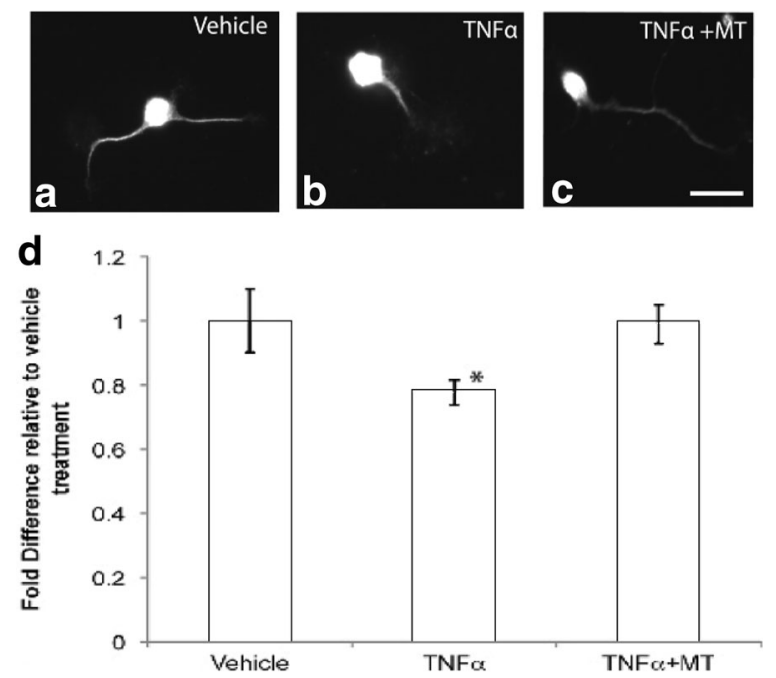

Fig. 2 Microglial inflammatory response was inhibitory to axonal outgrowth and this could be modulated (or improved) by extracellular metallothionein-II (MT). a shows the immunohistochemistry of neurons in the co-culture using the SMI312 antibody. The neurons treated with TNFa has a shorter process (or axon) when compare to vehicle. The addition of MT to the TNFa treatment has restored this inhibitory effect to a similar level as that receiving vehicle treatment $(\mathbf{a}-\mathbf{c})$. $\mathbf{d}$ shows the quantitative measurement of the neurite length relative to the vehicle treatment. (Scale Bar in $\mathbf{a}=12 \mu \mathrm{m})\left({ }^{*} p<0.05\right.$ compared to vehicle treatment and TNFa + MT using ANOVA; $n=4$ experimental cultures per treatment group; error bars = standard errors of the mean fold difference relative to vehicle treatment)

To determine whether LRP1 is involved in the action of MTII upon cultured microglia, we employed siRNA against LRP1 in the microglia-neuron co-culture model. We first validated the selectivity of the siRNA against LRP 1 receptors in our previous publication [17]. The decreased expression of LRP1 receptors in the presence of siRNA was also confirmed using Western blot (Fig. 3c). We then examined the effect of knocking down LRP1 expression upon the ability of MTII to modulate inflammatory-activated microglia to promote neurite outgrowth. We found that the effect of MTII upon TNF $\alpha$-stimulated microglia was ablated with reduced LRP1 expression, suggesting that MTII exerts its effect through the LRP1 receptor in vitro (Fig. 3d).

\section{Discussion}

In this study, we first demonstrated that the majority of the exogenously administered $\mathrm{MT}_{488}$ and $\mathrm{A}_{488}$ associated with the Iba1-labelled microglia/macrophages within close proximity to the needlestick injury in the cortex. The association of both the $\mathrm{MT}_{488}$ and $\mathrm{A}_{488}$ may be in accordance with the well-described function of microglia to phagocytose foreign material [18]. Using this fluorescence-labelled protein model, we can only demonstrate the association of MTII to microglia in vivo. A further study that involves injecting unlabelled MTII into a transgenic MT-null mice model will be needed to confirm the fate of the injected MTII via post-mortem immunolabelling. However, from what we observed using the fluorescence-labelled MTII, the microglia that had associated with $\mathrm{MT}_{488}$ had a ramified phenotype in comparison to those internalising $\mathrm{A}_{488}$. Previous studies have suggested the presence of two subtypes of microglia in the CNS; however, recent studies have suggested that these two subtypes could potentially be extremes of a spectrum between the neuro-toxic (M1) to neuro-protective roles (M2) that microglia have in response to an extracellular signal [19]. The changes in microglia phenotype in the presence of MTII observed in this study indicate that MTII plays a role in altering the function of the microglia to a more neuro-protective form rather than a switch in the subtypes. However, further study is needed to clarify the expression of the neuroprotective phenotype markers in the microglia of the brains treated with $\mathrm{MT}_{488}$. It should be noted in this experiment model, the amount of MTII injected $(1 \mu \mathrm{L}$ of $2 \mathrm{mg} / \mathrm{mL}$ of MTII) is not a significant amount compared to the endogenous MTII concentration in human. The concentration of the MTII used in this experiment model is mainly to allow us to study the cellular association of extracellular MTII, which is the main aim of this experiment. A limitation of this study is the use of the Iba-1 antibody, which labelled both microglia and infiltrating peripheral macrophages; this makes it difficult to specify whether the cells that have internalised the $\mathrm{A}_{488}$ and $\mathrm{MT}_{488}$ are microglia or infiltrated macrophages or both. To further investigate the direct influence that MTII has on microglia, we utilised a microglia-neuron co-culture model. In this co-culture model, the microglia is preconditioned with either TNF $\alpha$ only or TNF $\alpha$ and MTII in serum-free media, which is then replaced with neurobasal media containing $10 \%$ serum prior to the plating of cortical neurons; therefore, the addition of the serum-derived MTII will not affect the pre-conditioning on microglia from the treatments, hence the experiment outcome from the co-culture model. Using this model, we have demonstrated that pre-treating the microglia with TNF $\alpha$ (a proinflammatory cytokine) led to a decrease in neurite outgrowth and that the addition of MTII abrogated this effect. Hence, this demonstrated that MTII is capable of exerting a specific effect upon TNF $\alpha$-treated microglia in culture. It has been recently demonstrated that TNF $\alpha$ treatment in primary microglia culture leads to an inflammatory response via suppressing the expression LRP1 [20]. The activation of LRP1 receptors has been shown in various studies to be involved in decreasing the inflammatory response of microglia. It has also been demonstrated that MTII bind to both LRP1 and LRP2 (also known as megalin) receptors in neurons $[9,10,21]$. In this study, we hypothesised that MTII bind to LRP1 receptor on microglia 

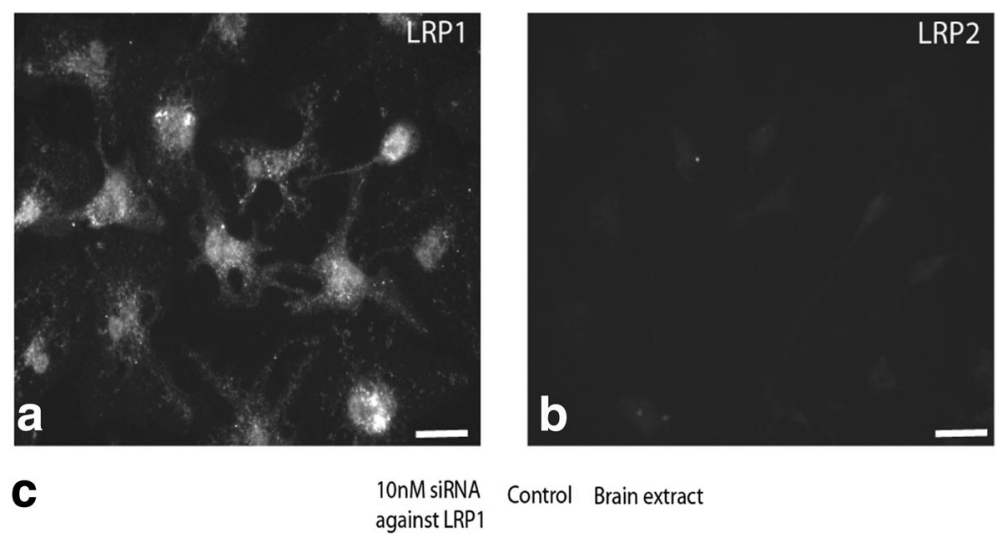

Control Brain extract against LRP1
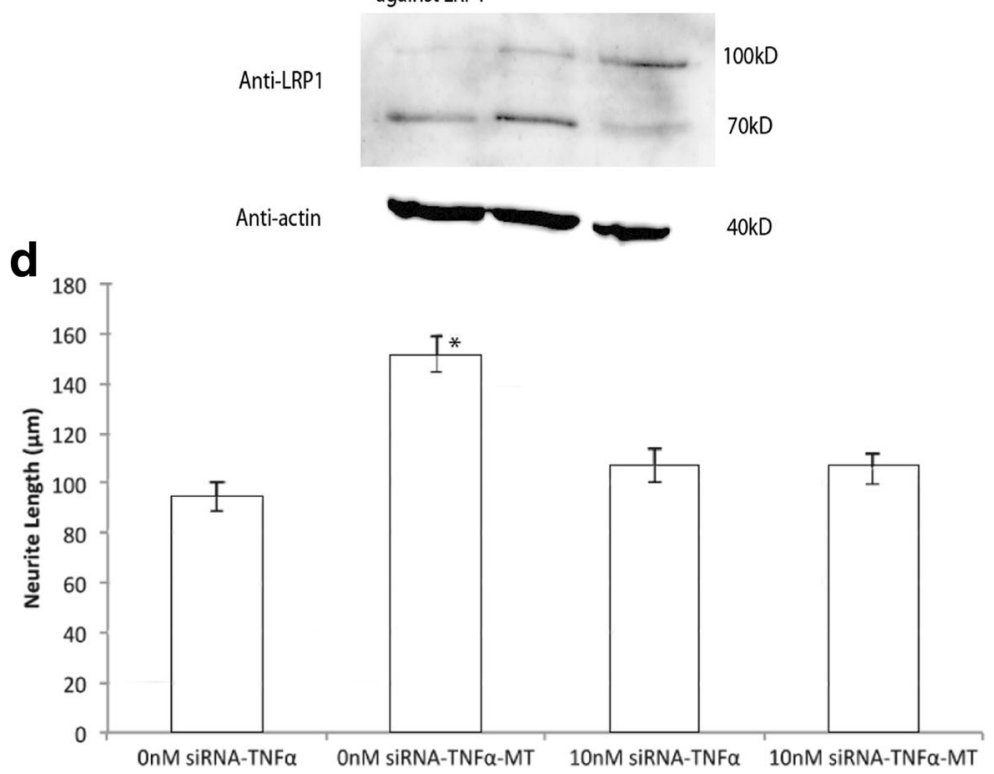

Fig. 3 Metallothionein II (MT) alter the TNFa-treated microglia response via LRP1 receptors in microglia and neuron co-culture model. Immunohistochemistry labelling using antibodies against LRP1 and LRP2 demonstrated that cultured microglia express LRP1 but not LRP2 receptors (a and b). c shows the Western blot performed on the protein extracted from microglia culture receiving $10 \mathrm{nM}$ siRNA or no siRNA. In comparison to the no siRNA control, there is a decrease in the expression of LRP1 (both 100kD and 70kD band). The brain extract from rat cortex was also used as an internal positive control for this western blot. $\mathbf{d}$ shows the quantitative results from the microglia-neuron co-culture with the treatment of siRNA against LRP1 added to the microglia in conjunction with the treatments (TNFa or TNFa + MT). Similar to the result shown previously, in cultures not receiving the siRNA, the neurite length was higher in the microglia pre-treated with MT and TNFa when compare to the treatment with TNFa. The addition of siRNA against LRP1 in the TNFa-treated microglia did not led to significant changes in the neurite length when compared to the TNFa treatment without the siRNA. In contrast, the addition of siRNA against LRP1 did not significantly increase the neurite length. (Scale bar at Figure $A=25 \mu \mathrm{m})\left({ }^{*} p<0.05\right.$ compared to other treatment; $n=4$ experimental cultures per treatment group, ANOVA; error bars = standard errors of the mean neurite length)

which leads to a decrease in the inflammatory response in microglia through its action on LRP1 receptors. To investigate this, we have utilised siRNA to knockdown LRP1 receptors in our microglia and neuron co-cultures. Our results demonstrated that the knockdown of LRP1 receptor attenuates the effect of MTII in promoting neurite outgrowth after TNF $\alpha$ treatment. This suggests that MTII modulates microglia function via the LRP1 receptor.

This is the first report showing that MTII acts through the LRP1 receptor to alter the microglia response. It has been previously reported that LRP1 receptor activation by an Apolipoprotein-E (Apo-E) mimetic peptide also decreases the microglia inflammatory response via a mitogen-activated protein kinase (MAPK) dependent pathway [22]. Notably, we have reported previously that MTII stimulates the growth of cultured cortical neurons via LRP2 or the megalin receptor $[9,10]$. Collectively, this suggests that LRP signalling could be involved in promoting axon regeneration and neural recovery following traumatic injury via multiple cellular pathways. We have previously reported that MTII modulates the behaviour of reactive astrocytes through a JAK/STAT signalling pathway [11]. A recent study had also demonstrated the involvement of LRP1 in microglia activation via modulating the JNK and NF- 
kappaB signalling pathways [20]. The JAK/STAT, JNK and NF-kappaB have been demonstrated to be important stress kinase pathways involved in microglia inflammatory response [23]. Thus, these pathways could potentially be the downstream pathway for MTII-LRP1 interaction in microglia; however, this will need to be confirmed in future studies.

In this current study, MTII treatment caused a distinct change in the morphology of microglia. In saline-treated animals, microglia displayed a spherical and amoeboid morphology that is proposed to be associated with a reactive and inflammatory phenotype that inhibits neural regeneration (commonly referred to as the M1 phenotype) [24]. However, MTII treatment caused a clear difference in microglial morphology, resulting in ramified structures that resemble the M2 phenotype that supports neural regeneration [24]. Our in vitro studies support the observation that MTII induces a pro-regenerative microglial phenotype in response to TNFo. Collectively, our data clearly demonstrates that MTII activates an LRP1 receptor-dependent pathway in microglia, providing for the first time a mechanistic explanation for the numerous in vivo reports that MTII modulates the microglial response to CNS injury and stress $[12-14,16]$.

\section{Conclusions}

In conclusion, this study has presented data supporting the neuroprotective role of MTII via modulating the microglia inflammatory response. This study has also identified LRP1 as a receptor for MTII in microglia, which further demonstrates the importance of LRP1 receptors in modulating the microglial response to promote axonal growth after injury. This study has demonstrated that in addition to the direct role that MTII exerts on neurons to promote their regeneration, MTII also indirectly promotes the regeneration (or recovery) of the damaged neurons by improving the extracellular environment (glial response) to support the axon regeneration after injury.

\section{Abbreviations \\ Apo-E: Apolipoprotein-E; CNS: Central nervous system; CSPG: Chondroitin sulphate proteoglycans; DMEM: Dulbecco's modified eagle medium;

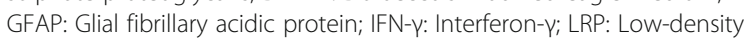 lipoprotein receptor; MAG: Myelin-associated glycoprotein; MAPK: Mitogen- activated protein kinase; MS: Mouse; MT: Metallothionein; \\ OMgp: Oligodendrocyte myelin glycoprotein; PBS: Phosphate buffer saline; QUIN: Quinolinic acid; RB: Rabbit; TNFa: Tumor necrosis factor-a}

\section{Acknowledgements}

Not applicable

\section{Funding}

The first author (Dr. Leung) is supported by the Bill Gole Postdoctoral Research Fellowship from the Motor Neuron Disease Research Institute of Australia (MNDRIA). This project is also supported by the Research Enhancement Grant Scheme, Faculty of Health, University of Tasmania.

Availability of data and materials

Not applicable

\section{Authors' contributions}

JYKL and RSC are involved in the study design and carried out the experiment for animal injury model in this manuscript. JYKL and WRB carried out the cell culture and the biochemical and immuno-labelling in this manuscript. JYKL, RSC and AEK are involved in the manuscript draft. All authors read and approved the final version of this manuscript.

\section{Ethics approval}

All animal experimentation was performed under the guidelines stipulated by the University of Tasmania Animal Ethics Committee, which is in accordance with the Australian code of practice for the care and use of animals for scientific purposes. (Ethics number: A11957)

Consent for publication

Not applicable

\section{Competing interests}

All authors declare that they have no competing interests.

\section{Publisher's Note}

Springer Nature remains neutral with regard to jurisdictional claims in published maps and institutional affiliations.

\section{Author details}

${ }^{1}$ Wicking Dementia Research and Education Centre, College of Health and Medicine, University of Tasmania, Medical Science Precinct 1, 17 Liverpool Street, Hobart, TAS 7000, Australia. ${ }^{2}$ Department of Biomedical Sciences, Faculty of Medicine \& Health Sciences, Macquarie University, 2 Technology Place, Sydney, NSW 2109, Australia.

Received: 6 July 2017 Accepted: 18 January 2018

Published online: 22 February 2018

References

1. Sofroniew MV. Molecular dissection of reactive astrogliosis and glial scar formation. Trends Neurosci. 2009;32(12):638-47.

2. D'Avila JC, Lam TI, Bingham D, Shi J, Won SJ, Kauppinen TM, Massa S, Liu J, Swanson RA. Microglial activation induced by brain trauma is suppressed by post-injury treatment with a PARP inhibitor. J Neuroinflammation. 2012;31(12):638-47.

3. Bradbury EJ, Moon LDF, Popat RJ, King VR, Bennett GS, Patel PN, Fawcett JW, McMahon SB. Chondroitinase ABC promotes functional recovery after spinal cord injury. Nature. 2002;416:636-40.

4. Chen XR, Liao SJ, Ye LX, Gong Q, Zeng JS, Yu J. Neuroprotective effect of chondroitinase $A B C$ on primary and secondary brain injury after stroke in hypertensive rats. Brain Res. 2014;1543:324-33.

5. Ji K, Tsirka SE. Inflammation modulates expression of laminin in the central nervous system following ischemic injury. J Neuroinflammation. 2012;6:159-70.

6. Horner PJ, Gage FH. Regenerating the damaged central nervous system. Nature. 2000;407:963-70

7. Fitch MT, Silver J. CNS injury, glial scars, and inflammation: inhibitory extracellular matrices and regeneration failure. Exp Neurol. 2008;209(2):294-301.

8. Yiu G, He Z. Glial inhibition of CNS axon regeneration. Nat Rev Neurosci. 2006:7(8):617-27.

9. Giger RJ, Hollis ER II, Tuzaynski MH. Guidance molecules in axon regeneration. Cold Spring Harb Perspect Biol. 2010;2(7):a001867.

10. Chung RS, Penkowa M, Dittmann J, King CE, Bartlett C, Asmussen JW, Hidalgo J, Carrasco J, YKJ L, Walker AK, Fung SJ, Dunlop SA, Fitzgerald M, Beazley LD, Chuah MI, Vickers JC, West AK. Redefining the role of metallothionein within the injured brain. Extracellular metallothionein play an important role in the astrocytes-neuron response to injury. J Biol Chem. 2008;283:15349-58.

11. Leung YKJ, Pankhurst M, Dunlop SA, Ray S, Dittmann J, Eaton ED, Palumaa $P$, Sillard R, Chuah MI, West AK, Chung RS. Metallothionein induces a regenerative reactive astrocyte phenotype via JAKJSTAT and RhoA signalling pathways. Exp Neurol. 2010;221:98-106

12. Chung RS, Leung YK, Butler CW, Chen Y, Eaton ED, Pankhurst MW, West AK, Guillemin GJ. Metallothionein treatment attenuates microglia activation and expression of neurotoxic quinolinic acid following traumatic brain injury. Neurotox Res. 2009;15:381-9. 
13. Giralt M, Penkowa M, Lago N, Molinero A, Hidalgo J. Metallothionein-1+2 protect the CNS after a focal brain injury. Exp Neurol. 2002;173:114-28.

14. Penkowa M, Carrasco J, Giralt M, Moos T, Hidalgo J. CNS wound healing is severely depressed in metallothionein I- and I-deficient mice. J Neurosci. 1999;19(7):2535-45.

15. Chung RS, Vickers JC, Chuah MI, West AK. Metallothionein-IIA promotes initial neurite elongation and postinjury reactive neurite growth and facilitates healing after focal cortical brain injury. J Neurosci. 2003;23(8):3336-42

16. Guillemin GJ, Smith DG, Smythe GA, Armati PJ, Brew BJ. Expression of the kynurenine pathway enzymes in human microglia and macrophages. Adv Exp Med Biol. 2003;527:105-12.

17. Landowski LM, Pavez M, Brown LS, Gasperini R, Taylor BV, West AK, Foa $\mathrm{L}$. Low-density lipoprotein receptor-related proteins in a novel mechanism of axon guidance and peripheral nerve regeneration. J Biol Chem. 2016;291:1092-102.

18. Lalancette-Hébert M, Gowing G, Simard A, Weng YC, Kriz J. Selective ablation of proliferating microglial cells exacerbates ischemic injury in the brain. J Neurosci. 2007;27(10):2596-605.

19. Ransohoff RM. A polarizing question: do M1 and M2 microglia exist? Nat Neurosci. 2016;19(8):987-91.

20. Yang L, Liu CC, Zheng $H$, Kanekiyo $T$, Atagi $Y$, Jia L, Wang D, N'songo A, Can $D, X u H$, Chen XF, Bu G. LRP1 modulates the microglial immune response via regulation of JNK and NF-kB signalling pathways. J Neuroinflammation 2016;13:304-316

21. Ambjorn M, Asmussen JW, Lindstam M, Gotfryd K, Jacobsen C, Kiselyov W, Moestrup SK, Penkowa M, Bock E, Berezin V. Metallothionein and a peptide modelled after metallothionein, EmtinB, induce neuronal differentiation and survival through binding to receptors of the low-density lipoprotein receptor family. J Neurochem. 2008;104:21-37.

22. Pocivavsek A, Mikhailenko I, Strickland DK, Rebeck GW. Microglial lowdensity lipoprotein receptor-related protein 1 modulates C-Jun N-terminal kinase activation. J Neuroimmunol. 2009:214(1-2):25-32.

23. Kacimi R, Giffard RG, Yenari MA. Endotoxin-activated microglia injure brain derived endothelial cells via NF-kB, JAK-STAT and JNK stress kinase pathways. J Inflamm. 2011;8:7-21.

24. Tang $Y$, Le $W$. Differential roles of $M 1$ and $M 2$ microglia in neurodegenerative diseases. Mol Neurobiol. 2016;53:1181-94

\section{Submit your next manuscript to BioMed Central and we will help you at every step:}

- We accept pre-submission inquiries

- Our selector tool helps you to find the most relevant journal

- We provide round the clock customer support

- Convenient online submission

- Thorough peer review

- Inclusion in PubMed and all major indexing services

- Maximum visibility for your research

Submit your manuscript at www.biomedcentral.com/submit 